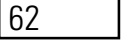

TECHNIQUES AND DEVICES FOR NEONATAL RESUSCITATION

doi:10.1136/archdischild-2012-302724.0062

1.2GM Schmölzer. 'Department of Pediatrics, University of Alberta, Edmonton, $A B$, Canada; '2Department of Paediatrics, Medical University of Graz, Graz, Austria

Establishing breathing and oxygenation after birth is vital for survival and long-term health of premature infants. However, approximately $10 \%$ of premature infants require breathing support at birth. An international consensus and various national resuscitation guidelines suggest techniques and equipment for neonatal resuscitation. They all agree positive pressure ventilation is the cornerstone of breathing support in the delivery room.

A lung-protective strategy should start immediately after birth. To facilitate the early development of functional residual capacity, and improve oxygenation during the transition of preterm infants, sustained inflations, positive end expiratory pressure and continuous positive airway pressure (CPAP) should be applied at the initiation of respiratory support. Although sustained inflations (SI) are advocated as lung recruitment maneuvers and positive end expiratory pressure helps to maintain end expiratory lung volume, neither of these has been mandated in neonatal resuscitation guidelines. This presentation will provide an update on current literature about techniques and devices used during neonatal resuscitation. Initial respiratory support provided with either CPAP, SI, and positive pressure ventilation along with available devices (e.g. face mask, nasal prong, Guedel airway, Laryngeal airway mask) will be discussed. In addition, new insights about intubation and chest compressions will be presented.

\section{ADULT OUTCOMES IN ACHIEVEMENT AND SOCIAL INTEGRATION}

doi:10.1136/archdischild-2012-302724.0063

P Anderson. Critical Care \& Neurosciences, Murdoch Children's Research Institute, Parkville, VIC, Australia

The lecture will provide a wide overview of studies on achievements as indicated by academic and occupational outcome and independent living of those born preterm. The lecture will further explore how well preterm children are integrated into society, participate in peer activities, have formed partner relationships and are reproductively active. How do they view their quality of life? Most of these studies have focussed on very preterm or very low birth weight infants but where available, information on moderate to late preterm adults will also be addressed.

\section{WE THINK WE STILL HURT OUR NEWBORNS!}

doi:10.1136/archdischild-2012-302724.0064

${ }^{1} \mathrm{~A}$ Bos, 'D Roofthooft, ${ }^{1,2} \mathrm{M}$ van Dijk. 'Neonatal Intensive Care; ${ }^{2}$ Intensive Care, Erasmus MC - Sophia Children's Hospital, Rotterdam, The Netherlands

Background and aims Painful procedures in Neonatal Intensive Care Units are hard to avoid. We documented a drop in painful procedures from average 14 in 2001 to 12 in 2009 per patient per day. This study aimed to uncover how nursing and medical staff perceive the painfulness of these procedures and if perceptions have changed over time.

Methods Both in 2001 and 2009 nursing and medical staff rated the painfulness of 15 skin-breaking and 13 other procedures on a scale from 0 (not painful) to 10 (worst possible pain). The ratings of skin-breaking and other procedures served as outcome measures.

Results Response rates were $60 \%(\mathrm{~N}=58)$ and $72.4 \% \quad(\mathrm{~N}=84)$ respectively in 2001 and 2009 . The mean rating of the skin-breaking procedures decreased from 6.5 (SD 1.4) in 2001 to 6.1 (SD 1.5) in $2009(\mathrm{p}=0.18)$. The mean rating of other skin-breaking procedures decreased from 4.8 (SD 1.3) to 4.5 (SD 1.3) ( $p=0.25$ ). Contrary to the trend, chest tube insertion was rated statistically significantly higher by nurses and physicians in 2009, $p<0.001$ and $p=0.004$ (Figure).

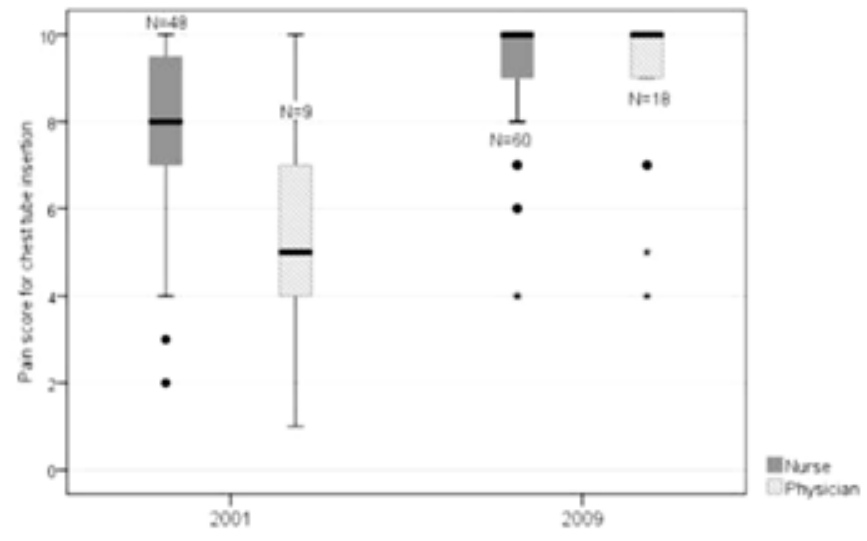

Abstract 64 Figure 1

Conclusion Overall, perceptions of health professionals in our Neonatal ICU concerning painfulness of procedures have not changed significantly over time despite the introduction of pain reducing interventions such as sucrose and developmental care after 2001.

\section{COMPARISON OF EFFECT OF KANGAROO CARE AND MOTHER HOLDING ON SLEEP AND WAKING STATES OF PRETERM NEONATES}

doi:10.1136/archdischild-2012-302724.0065

N Rajai, F Bastani, E Amini, H Haghani, S Janmohammadi. Tehran University of Medical Sciences, Faculty of Nursing and Midwifery, Tehran, Iran

Background and aim Sleep and waking disorders are the main problems of preterm neonates in Neonatal Intensive Care Unit (NICU) that occur influence by many stimuli and care activities of NICU and this may have irreversible effects on their brain development. So that the objective of this study is to assess the effect of two care methods on the sleep and waking states of preterm neonates.

Method In a clinical trial study a total of 70 eligible preterm neonates with gestational age between 32-37 weeks and admitted to NICU were selected and randomly divided in two groups of kangaroo care and holding by mother without directly skin contact. Data collection process was carried out using the behavioral sleep and wake scale of Als. The researcher every two minutes observed and recorded the sleep and wake behavior of neonates of two groups, in 20 minutes pre intervention, during 70 minutes of intervention and during 20 minutes post intervention. The collected data were analyzed using variance analysis test via repetitive sizes and Independent $\mathrm{T}$ test.

Result The kangaroo care group in compare to holding group significantly had more quiet sleep and alert awake and less drowsiness $(\mathrm{P}<0 / 001)$, active awake, and crying states $(\mathrm{P}=0 / 002)$.

Conclusion Neonates had more beneficial and less undesirable states of sleep and waking during kangaroo care. So that kangaroo care may be helpful to improve sleep and waking states of preterm neonates in NICU. 
1.2R Maastrup. 'Knowledge Centre for Breastfeeding Infants with Special Needs/NICU, Rigshospitalet, Copenhagen, Denmark; ${ }^{2}$ Department of Health Sciences, Faculty of Medicine, Lund University, Lund, Sweden

Background Breastfeeding of preterm infants is not well researched in Denmark, although breast milk is of great importance for these infants because it protects against infections, decrease the risk for severe diseases (necrotizing enterocolitis and retinopathy), and breastfeeding strengthens bonding and attachment between mother and infant. Many factors can influence breastfeeding of preterm infants. Some are related to the mother and infant, while some are related to unit-specific factors at the Neonatal Intensive Care Unit (NICU). This study will highlight potential influencing factors.

Aim To investigate how many preterm infants are breastfed exclusively at discharge, and breastfeeding duration. To examine which conditions in infants, mothers and NICUs that affect breastfeeding of preterm infants.

Method A multicenter exploratory longitudinal survey on breastfeeding of preterm infants in all NICUs in Denmark with questionnaires answered by the mothers in beginning of hospitalisation and at the infants' discharge, followed by telephone interviews in the infants' first year.

Preliminary results More than 1400 preterm infants from 24 to 36 weeks of gestational age (mean 33 weeks and 4 days, SD 249 weeks) participated in the study. At discharge $68 \%$ were exclusively breastfed, $17 \%$ were partially breastfed and $15 \%$ were not breastfed. Further analysis will show which factors in infants, mothers and NICUs influence breastfeeding at discharge.

Conclusion Compared to most international research, preterm infants in Denmark have high breastfeeding rates at discharge from NICU.

\section{THE EXPERIENCE OF NURSES TAKING CARE OF NEWBORNS WITH NEONATAL ABSTINENCE SYNDROME (NAS) AND THEIR FAMILIES}

doi:10.1136/archdischild-2012-302724.0067

'AP França, ${ }^{2}$ SM Pereira, 'MC Reisinho. 'UNIESEP (Oporto College of Nursing Resaearch Unit), Oporto College of Nursing, Porto; ${ }^{2}$ Unidade de Cuidados Especiais Pediátricos/ Neonatais, Hospital Santo Espírito de Angra do Heroísmo-EPER, Angra do Heroísmo, Portugal

This study entitled "The experience of nurses taking care of newborns with Neonatal Abstinence Syndrome (NAS) and their families" gives an account of the nurse's experiences on a Neonatal Intensive Care Unit (NICU), as well as the difficulties they face regarding this matter. This is a qualitative, exploratory and descriptive study, with a phenomenological approach. Data was collected by semi-structured interviews and submitted to content analysis (Bardin). The analyses brought out the following themes: how the nurse perceives the child and the parents he/she is taking care; how the nurse perceives the way that parents take care of their child; what the nurse feels when he/she is taking care of the child and the family; the difficulties the nurse has to face; what the nurse focuses his/her attention when taking care of the child and the family; what the nurse perceives as necessary to improve the care which is being provided.

Nurses expressed some difficulties concerning the teamwork, difficult relationship and communication with the families, and lack of resources, of a specific training and care standards, as well as an updated assessment tool. They feel frustration, anxiety, revolt, weariness, anguish, pain, as well as affection, satisfaction, challenge and recognition. In general, parents are absent and disinterested. However some are more present and provide basic care for their child autonomously.

This study has revealed the complexity and importance of taking care of such children, associated with their characteristics, clinical manifestations, suffering and peculiar characteristics of their families.

\section{PSYCHOLOGICAL SYMPTOMS OF ADOLESCENTS WITH AND WITHOUT CHRONIC ILLNESSES}

doi:10.1136/archdischild-2012-302724.0068

${ }^{1}$ A Ergun, ${ }^{2} S$ Topçu, ${ }^{2} S$ Albayrak, ${ }^{2} 1$ Isik. ${ }^{1}$ Community Health Nursing Department, Marmara University, Faculty of Health Science, Division of Nursing; ${ }^{2}$ Koc University, School of Nursing, Istanbul, Turkey

Background and aims The aim of this study was to compare psychological symptoms of adolescents with and without chronic illnesses.

Methods A total of 380 students ( 255 healthy and 85 with chronic illnesses) were recruited for the study. Data were gathered by using the general information form and the brief symptom inventory (BSI) for adolescent. Mann-Whitney-U test were used in analyzing the information.

Results Results demonstrated that adolescents with chronic illnesses had higher scores on somatization $(p<0.001)$ and lower scores on hostility $(p<0.05)$. Girls with chronic illnesses had lower scores on depression $(p<0.01)$, negative self-perception $(p<0.05)$ and hostility $(p<0.01)$ than healthy girls. Both girls and boys with chronic illnesses had higher somatization scores than peers without chronic illnesses.

Conclusions This research suggests the importance of mental health screening for adolescents with chronic illnesses and the need for clinical and community-based interventions to target modifiable lifestyle factors that contribute to psychological distress.

\section{FATIGUE IN CHILDREN BEFORE AND AFTER BONE MARROW TRANSPLANTATION: A PRELIMINARY DATA}

doi:10.1136/archdischild-2012-302724.0069

'F Petrangeli, 'F D'Agostino, 'A Sili, 2 T Petrangeli, 'S Alessandrini, 'R Alvaro. 'School of Nursing, University 'Tor Vergata'; 'Department of Pediatric Hematology and Oncology, Bambino Gesù Children's Hospital, IRCCS, Rome, Italy

Background and aims Fatigue is described as one of the most frequent and distressing symptoms of cancer therapy but in children and adolescents it has received limited clinical attention. The aim of the study was to assess the change in fatigue scores during cancer treatment according to children's, adolescents' and parents' perspectives and to describe the possible causes of fatigue.

Methods In this longitudinal descriptive study were enrolled 21 patients (aged8-18 years) 15(71\%) boys and 6(29\%) girls, with acute lymphoblastic (ALL66.6\%) and myeloid (AML33.4\%) leukemia, and one of their parents. Fatigue as measured by PedsOL Multidimensional Fatigue Scale the day before the first cycle of chemotherapy (T0) and after 30 days (T1) of induction chemotherapy (about 25 days after BMT).

Results Compared data pre and post chemotherapy children had significantly worse fatigue total scores (79.3 DS $\pm 10.5 \mathrm{vs} 42.9 \mathrm{DS} \pm 8.5$, $\mathrm{p}=0.000)$ : general fatigue domain (77.4DS $\pm 14.4 \mathrm{vs} 31.3 \mathrm{DS} \pm 10.1$ $\mathrm{p}=0.000$ ), sleep/rest fatigue domain $(73.4 \mathrm{DS} \pm 15.8 \mathrm{vs} 28.6 \mathrm{DS} \pm 12.1$, $\mathrm{p}=0.000$ ), and cognitive fatigue was the better and similar domain pre and after BMT in child's (87.1DS \pm 11.2 vs69DS $\pm 17.5, p=0.000$ ) and parents' (88.3DS+12vs68.2DS \pm 21.4 ) perception. Children with ALL (41.7DS \pm 8.9 ) had a worse fatigue than AML (45.6DS \pm 7.6 ) and the fatigue parent's perception about their children is worse than child's perception (38.2vs42.9).

Conclusions This study shown that cancer treatment increase significantly children's fatigue levels. Nurses should frequently assess fatigue in children receiving chemotherapy and apply timely and tailored interventions to match the factors that contribute to fatigue and influence fatigue severity, further to identify the domains compromises and make correct nursing diagnoses. Management of fatigue during treatment will help children stay involved in age-related activities and meet developmental milestones. 